\title{
Efecto del suplemento de aceites vegetales sobre el perfil lipídico en ratas Wistar
}

\author{
Elpidia Poveda ${ }^{1}$, Paola Ayala ${ }^{1}$, Milena Rodríguez ${ }^{1}$ Edgar Ordóñez ${ }^{1}$, Cesar Baracaldo ${ }^{1}$ \\ Willman Delgado ${ }^{2}$, Martha Guerra ${ }^{3}$ \\ ${ }^{1}$ Grupo de Nutrición, Subdirección de Investigación, Instituto Nacional de Salud, Bogotá, D.C., Colombia \\ ${ }^{2}$ Centro de Investigaciones en Palma de Aceite, Cenipalma, Bogotá, D.C., Colombia. \\ ${ }^{3}$ Grupo de Investigación Clínico-Genético-Molecular en Dislipoproteinemias, Departamento de Ciencias \\ Pontificia Universidad Javeriana, Bogotá, D.C., Colombia.
}

Introducción. Se informan efectos sobre el perfil lipídico por acción de los tocoferoles, tocotrienoles y ácidos grasos saturados, monoinsaturados y poliinsaturados.

Objetivo. Evaluar el efecto del consumo de aceites vegetales de alta utilización en Colombia (palma, soya, maíz, girasol y canola) sobre las concentraciones sanguíneas de triglicéridos, colesterol total y colesterol-HDL en ratas Wistar.

Metodología. Se cuantificaron tocoferoles, tocotrienoles y ácidos grasos de los aceites por cromatografía líquida de alta resolución. A los animales se les suministró un suplemento de 0,2 $\mathrm{ml} /$ día de aceite durante 4 semanas; se sacrificó un grupo de cada tratamiento (grupos tratados con aceite de palma, aceite de soya, aceite de maíz, aceite de girasol y aceite de canola) para obtener muestras de sangre y cuantificar triglicéridos, colesterol total y colesterolHDL. Los datos se analizaron según media, desviación estándar, análisis de varianza y Bonferroni.

Resultados. No se presentaron diferencias en los triglicéridos a excepción del grupo control versus soya en la tercera semana de tratamiento; se observó también una tendencia a la disminución en el grupo de palma y al aumento en los de girasol y canola. No se encontraron diferencias significativas en colesterol total en ninguna de las semanas de intervención. Se presentaron diferencias en las concentraciones de colesterol HDL en las semanas de tratamiento $(p=0,005)$, una tendencia a la disminución en el grupo de palma y al aumento en el grupo de girasol y maíz.

Conclusión. Los aceites modifican el perfil lipídico; el bajo contenido de ácidos grasos saturados, el contenido de tocoferoles y tocotrienoles son favorables para el aumento del colesterol HDL; los tocotrienoles probablemente disminuyen los troglicéridos y atenúan las respuestas desfavorables de los ácidos grasos saturados.

Palabras clave: ratas/lipidosis, perfil lipídico, aceites vegetales, colesterol HDL, triglicéridos, vitamina $\mathrm{E}$, tocoferoles, tocotrienoles, suplementos dietéticos.

\section{Effects of vegetal oil supplementation on the lipid profile of Wistar rats}

Background. Dietary tocopherols, tocotrienols and saturated, mono and polyunsaturated fatty acids have been reported to have an effect on blood lipid profiles. In Colombia, vegetable oils (palm, soy, corn, sunflower, and canola) are a common dietary constituent and consumed in high quantities.

Objective. In the current study, the effects of vegetable oil consumption was examined by measuring blood concentrations of triglycerides (TG), total cholesterol (TC) and HDL cholesterol (HDL-C) in male Wistar rats.

Methods. The concentrations of tocopherols, tocotrienols, and fatty acids in each oil was quantified by High Performance Liquid Chromatography (HPLC). Each rat diet was supplemented with $0.2 \mathrm{ml} /$ day with one oil type. Over a 4-week period, groups of animals were sacrificed weekly and blood samples were obtained to quantify TC, TG and HDL-C for each oil class. Statistical analyses included mean, standard deviation, ANOVA and Bonferroni comparisons tests. 
Results. Triglyceride content was not affected except in the control and the soy group in the third treatment week, although a tendency for decreased TG was noted in the palm oil group and for increased TG in the sunflower oil and canola oil groups. No significant differences in total cholesterol were observed. In HDL-C, significant differences were present for every treatment week $(p=0.005)$; this represented a decreasing trend in palm oil group and an increasing trend in the sunflower and corn oil groups.

Conclusion. The oils effected changes in the blood lipid profile. A small amount of saturated fatty acids (tocopherol and tocotrienol) were favourable for the HDL-C increase. The presenct of tocorienols tended to decrease the TG and probably helped attenuate the unfavorable effects of the saturated fatty acids.

Keywords: rats, blood lipid profile, vegetable oils, total cholesterol, HDL cholesterol, triglycerides, vitamin E, tocopherols, tocotrienols.

La vitamina $E$ es un nutriente liposoluble que actúa como antioxidante natural cuando reacciona con los radicales libres solubles en los lípidos de membranas celulares; el efecto estabilizador sobre las membranas se observa en el metabolismo normal del oxígeno, en la peroxidación y en los trastornos del metabolismo producidos por algunas alteraciones patológicas (1-2). El $\alpha$ tocoferol es la forma biológica más potente $(100 \%) ;$ le siguen el $\beta$-tocoferol y, luego, el $\gamma$ y $\delta$ tocoferol; de los tocotrienoles, la forma activa es el $\alpha$-tocotrienol que proporciona estabilidad contra la oxidación (3) y en animales es un antioxidante y agente hipolipémico reductor del colesterol total más potente que los tocoferoles (4). Algunas de las funciones de los tocotrienoles son: inhibir la 3-hidroximetil-3-metilglutaril-coenzima A (HMGCoA) reductasa, reducir el colesterol total circulante, disminuir las concentraciones de colesterol LDL, promover la formación de prostaciclinas (antitrombóticas) e inhibir los tromboxanos (protrombóticos) al prevenir la agregación plaquetaria y reducción de la formación de trombos (5).

Las fuentes fundamentales de vitamina $E$ son los aceites de soya, maní, algodón, palma y girasol (6). La forma biológica tocotrienol se encuentra específicamente en el aceite de palma, en el salvado de arroz y en la cebada (7). Los estudios

Correspondencia:

Elpidia Poveda, Avenida Calle 26 No 51-60, zona 6, CAN, Bogotá, D.C., Colombia.

Teléfono: (571) 2207700 , extensiones 311 y 301; fax: (571)

2207700 , extensión 255

epoveda@ins.gov.co y epoveda1@yahoo.com

Recibido: 08/07/04; aceptado: 27/01/05 en animales alimentados con dietas que difieren en el tipo de aceite y en la concentración de tocoferoles y tocotrienoles muestran diversos efectos sobre el perfil lipídico. En ratas que reciben suplementos de aceite de canola se han encontrado mayores concentraciones séricas de colesterol HDL y menores de colesterol LDL (8); en animales alimentados con dietas altas en colesterol pero con suplemento de $\alpha$-tocoferol se han observado respuestas hipocolesterolémicas, protección contra modificaciones oxidativas del colesterol LDL e inhibición de la formación de la placa ateromatosa (9-11); en hámsters, el suplemento con tocotrienoles disminuye el colesterol total y el colesterol LDL en sangre. Los estudios también demuestran que el efecto sobre el perfil lipídico es más potente cuando el $\gamma$ tocotrienol se encuentra solo y no mezclado con otros tocotrienoles (12-14).

Los aceites vegetales, además de diferenciarse en las concentraciones y formas biológicas de vitamina $E$, también difieren en los ácidos grasos que los constituyen; estas diferencias generan distintos efectos en el perfil lipídico que se hacen más evidentes en las concentraciones de colesterol HDL (8). El aceite de palma, a pesar de ser rico en ácidos grasos saturados, reduce el colesterol total, el colesterol LDL y los triglicéridos, y aumenta las concentraciones de colesterol HDL cuando se ofrece con dietas equilibradas; este efecto se relaciona con su contenido de tocoferoles, tocotrienoles y $\beta$ carotenos $(15,16)$. Los ácidos grasos poliinsaturados de aceites vegetales inhiben la síntesis e incrementan el catabolismo del colesterol VLDL, disminuyen la síntesis de ApoB- 
100 y colesterol LDL, y disminuyen la lipemia posprandial (8).

En ratas que reciben un suplemento de diferentes aceites se han observado aumentos en el colesterol HDL y reducciones en las concentraciones de triglicéridos y colesterol total sólo en los grupos que han recibido un suplemento con aceite de canola. Este efecto al parecer se relaciona con el contenido de ácido $\alpha$-linoleico (1719). Los ácidos grasos monoinsaturados también pueden reducir las concentraciones de lípidos tan eficientemente como los poliinsaturados al disminuir las concentraciones de colesterol total, triglicéridos y colesterol $\operatorname{LDL}(20,21)$.

El estudio comparó el efecto del consumo de diferentes aceites vegetales de alto consumo y producción en Colombia, con diferentes concentraciones de vitamina $E$ (tocoferoles y tocotrienoles) y distinta composición de ácidos grasos sobre las concentraciones sanguíneas de colesterol total, colesterol HDL y triglicéridos en un modelo animal con ratas Wistar.

Algunos estudios mencionan que existe gran similitud entre el sistema cardiovascular de las ratas y otros mamíferos, incluido el hombre; esto la hace un modelo viable para evaluar el efecto de distintos aceites sobre los lípidos sanguíneos (22)

\section{Materiales y métodos}

\section{Aceites}

Se seleccionaron oleína de palma refinada blanqueada y desodorizada, aceites de soya, maíz, canola y girasol comúnmente consumidos en Colombia. Las soluciones oleosas se mantuvieron en frascos ámbar hasta su uso. Los tocoferoles, tocotrienoles y ácidos grasos de los aceites se cuantificaron por cromatografía liquida de alta resolución (HPLC) con un cromatógrafo Merck-Hitachi, serie LaChrom, equipado con bomba (modelo L-7100), inyector automático (modelo L-7200) y detector UV-Vis modelo (L7400) a una longitud de onda de $254 \mathrm{~nm}$, programa D-7000 v. 4,0 y columna Chromolith RP- $18 \mathrm{e} 100 \mathrm{x}$ 4,6 mm (Merck).

Las muestras de aceite se saponificaron en un tubo de ensayo con taparrosca, se pesaron $0,1 \pm 0,01 \mathrm{~g}$ de aceite previamente homogenizado, y se añadieron $0,25 \mathrm{ml}$ de una solución de $\mathrm{KOH}$ $2 \mathrm{~N}$ en metanol. El tubo se tapó herméticamente y se colocó en baño de María a $80 \pm 5^{\circ} \mathrm{C}$ durante 1 hora. Luego, se adicionaron $0,3 \mathrm{ml} \mathrm{de} \mathrm{H}_{2} \mathrm{SO}_{4} 2 \mathrm{~N}$. Los ácidos grasos liberados se extrajeron con hexano $(3 \times 1 \mathrm{ml})$ previa saturación de la fase acuosa con sulfato de sodio anhidro. El extracto orgánico obtenido se transfirió a un balón aforado de $5 \mathrm{ml}$ y se completó a volumen con hexano.

\section{Obtención de los ésteres de p-bromofenacilo}

Se tomó una alícuota de $0,5 \mathrm{ml}$ del extracto de ácidos grasos en un tubo de ensayo con taparrosca, y se adicionaron $0,9 \mathrm{ml}$ de una solución de 2,4'-dibromoacetofenona ( $12 \mathrm{mg} / \mathrm{ml}$ en acetona) y $0,9 \mathrm{ml}$ de una solución de trietilamina $(10 \mathrm{mg} / \mathrm{ml}$ en acetona). La mezcla se agitó mecánicamente (1 minuto), se tapó herméticamente y se colocó en baño de María a $60^{\circ} \mathrm{C}$ durante 2 horas. La solución obtenida se filtró a través de filtros PDVF de $0,45 \mathrm{~mm}$. Veinte $\mathrm{ml}$ de esta solución se inyectaron al cromatógrafo.

Los distintos isómeros de tocoferoles y tocotrienoles de la vitamina $\mathrm{E}$ se determinaron empleando el método del estándar externo, con patrones certificados de referencia de tocoferoles y tocotrienoles del 95\% de pureza (Calbiochem).

La composición de ácidos grasos y vitamina $\mathrm{E}$ de los aceites que se utilizaron se describe en el cuadro 1.

\section{Modelo animal y pruebas de laboratorio}

Se utilizó un modelo animal con ratas Wistar para establecer el efecto del consumo de los aceites en las concentraciones sanguíneas de colesterol total, colesterol HDL y triglicéridos. Para tener una mayor homogeneidad y disminuir la variabilidad biológica en los grupos, se utilizaron 96 ratas Wistar macho de cuatro semanas suministradas por el Instituto Nacional de Salud (INS) y con pesos de 60 a $80 \mathrm{~g}$ al inicio del ensayo (peso promedio $=75,6 \pm 9,7 \mathrm{~g}$ ). Los animales se dividieron en seis grupos teniendo en cuenta los cinco aceites que se iban a evaluar (palma, girasol, soya, maíz y canola), y un grupo control sin suplemento. Los animales se colocaron en cajas de policarbonato y se mantuvieron en el bioterio del INS bajo condiciones de humedad $(60 \pm 10 \%)$, 
Cuadro 1. Porcentaje de ácidos grasos y concentraciones de vitamina $E$ de los aceites vegetales usados para el suplemento.

\begin{tabular}{|c|c|c|c|c|c|}
\hline \multirow{2}{*}{$\begin{array}{l}\text { Ácidos grasos } \\
(\%)\end{array}$} & \multicolumn{4}{|c|}{ Aceites } & \multirow[b]{2}{*}{ Palma } \\
\hline & Girasol & Soya & Maíz & Canola & \\
\hline Saturados & 11,03 & 16,88 & 12,35 & 7,27 & 39,77 \\
\hline Monoinsaturados & 33,16 & 22,72 & 29,81 & 60,74 & 43,49 \\
\hline Poliinsaturados & 49,61 & 57,99 & 53,89 & 27,67 & 12,3 \\
\hline \multicolumn{6}{|l|}{ Vitamina E (ppm) } \\
\hline$\alpha$-tocoferol & 475,10 & 141,00 & 214,70 & 174,10 & 223,80 \\
\hline$\beta+\gamma$-Tocoferol & 31,40 & 825,60 & 453,60 & 424,50 & 31,20 \\
\hline$\delta$-tocoferol & - & 568,80 & 86,90 & 18,90 & 15,70 \\
\hline$\alpha$-tocotrienol & - & - & 27,10 & - & 310,90 \\
\hline$\beta+\gamma$-tocotrienol & - & _- & _ & - & 492,60 \\
\hline$\delta$-tocotrienol & - & - & - & - & 65,40 \\
\hline Total & 506,50 & 1535,40 & 782,30 & 617,50 & 1139,60 \\
\hline
\end{tabular}

luz (luz artificial de 6 a.m. a 6 p.m.) y temperatura $\left(21 \pm 1^{\circ} \mathrm{C}\right)$ controladas. Todos los grupos recibieron agua y el concentrado comercial para animales de laboratorio (Rodentina $\AA$ Purina) a libre demanda (con $10 \%$ de la composición en grasa).

Cada animal recibió un suplemento de 0,2 ml por día de aceite vegetal con una jeringa desechable de $1 \mathrm{ml}$, graduada en $0,01 \mathrm{ml}$, unida a una sonda nasogástrica de nelatón calibre 4 o 6 . Cada sonda se depuró con los mismos suplementos antes de realizar el procedimiento de alimentación forzada. La sonda se introdujo rápidamente por el esófago del animal hasta alcanzar el estómago para no producir ningún malestar en el animal. La sonda y la jeringa se cambiaron diariamente para evitar la contaminación de los animales o de los suplementos. Semanalmente, durante 4 semanas, se sacrificó un grupo de cuatro ratas de cada grupo de tratamiento para obtener las muestras de sangre. Diariamente se pesaron las ratas para verificar que el procedimiento no estuviera alterando el crecimiento normal ni las condiciones del animal.

Para obtener las muestras de sangre, los animales se anestesiaron con una mezcla de quetamina y xilasina y se extrajo un máximo de $5 \mathrm{ml}$ de sangre por punción cardiaca con jeringa desechable. El suero se separó por centrifugación dentro de las tres horas después de la toma de la muestra y se congelo a $-70^{\circ} \mathrm{C}$ hasta su procesamiento.

Las concentraciones de colesterol total y triglicéridos se determinaron utilizando estuches enzimáticos de Serapak-Bayer. El colesterol HDL se determinó en el sobrenadante después de centrifugación y tras la precipitación de las LDL con ácido fosfotúngstico e iones de magnesio.

\section{Métodos estadísticos}

Los datos de los tratamientos se describieron utilizando la media y la desviación estándar. Para evaluar las diferencias entre los valores de los indicadores bioquímicos del grupo control y los tratamientos, se aplico el análisis de varianza (ANOVA) y cuando existieron diferencias estadísticamente significativas se hicieron comparaciones entre medias 2 a 2 mediante la prueba de comparación de Bonferroni, el nivel para considerar diferencias estadísticamente significativas en ambas pruebas fue de $p<0,05$. Previo al uso de ANOVA se verificó la homogeneidad de las varianzas por la prueba de Levene $(p>0,05)$. En las variables que no tenían distribución normal se aplicó el Kruskall-Wallis por medio de la prueba asintótica y técnica de Monte Carlo (simulaciones) para compensar el efecto que sobre el Kruskall-Wallis en su cálculo asintótico pudiera tener el tamaño pequeño de la muestra.

La descripción numérica de los datos se complementó con procedimientos gráficos que permitieron visualizar su comportamiento; los gráficos se elaboraron en los sistemas Statistica, versión 5.5, y Power Point. Las bases de datos se crearon en Access y para el análisis estadístico se usó SPSS, versión 10. 


\section{Consideraciones éticas}

Los procedimientos se realizaron de acuerdo con las normas científicas, técnicas y administrativas para la investigación en salud (Resolución No. 8430 de 1993), la Ley 84 de 1989 y los principios éticos de la experimentación animal del International Council for Laboratory Animal Science. El estudio fue aprobado por el Comité de Ética e Investigación del Instituto Nacional de Salud de Colombia.

\section{Resultados}

La composición de ácidos grasos y vitamina $\mathrm{E}$ de los aceites que se utilizaron se describe en el cuadro 1. Se encontraron tocotrienoles en los aceites de palma y maíz, con mayor contenido en el de palma y, especialmente, de tipo $\alpha$ tocotrienol. La mayor concentración de $\alpha$-tocoferol se observó en el aceite de girasol, de $\beta-$ y $\gamma$ tocoferol en los aceites de soya y maíz y de $\delta$ tocoferol en los aceites de soya y maíz. En cuanto a los ácidos grasos, la mayor concentración de saturados se presentó en el aceite de palma, seguido por el aceite de canola. Los aceites de canola, palma, girasol y maíz presentaron la mayor concentración de ácidos grasos monoinsaturados; la mayor concentración de ácidos grasos poliinsaturados se identificó en los aceites de soya y maíz y la menor concentración de estos ácidos grasos, en el aceite de palma.

\section{Triglicéridos}

En la semana 1 no se observaron diferencias significativas en las concentraciones de triglicéridos entre los distintos tratamientos; no obstante, los grupos con suplementos de maíz y soya presentaron las mayores concentraciones. En la semana 2 tampoco se encontraron diferencias estadísticamente significativas entre las concentraciones de triglicéridos de los tratamientos, aunque el aceite de canola mostró la mayor concentración (cuadro 2).

En la semana 3 se observaron diferencias significativas entre los tratamientos; estas diferencias se detectaron entre el grupo control y el tratamiento con soya que mostró las mayores concentraciones; en los otros tratamientos las diferencias no fueron significativas. En la semana
4 no se identificaron diferencias en las concentraciones de triglicéridos pero los grupos con suplementos de aceite de girasol y de canola presentaron las mayores concentraciones (cuadro 2).

La figura 1 muestra el valor medio de las concentraciones de triglicéridos en las diferentes semanas y tratamientos; se observa una tendencia al aumento en las concentraciones de los grupos con suplementos de aceite de girasol y canola y de menores concentraciones en el grupo con suplemento de aceite de palma, el cual presentó, a excepción de la semana 3 , el menor valor y la menor variabilidad en todas las semanas de tratamiento.

\section{Colesterol total}

En la semana 1 no se observaron diferencias en las concentraciones de colesterol total entre los grupos de cada tratamiento. En las semanas 2, 3 y 4 tampoco se encontraron diferencias significativas entre tratamientos; sin embargo, en la semana 4 el grupo control obtuvo las menores concentraciones de colesterol total y el grupo con aceite de canola, las mayores concentraciones (cuadro 2); el grupo de canola mostró, además, una tendencia semanal hacia el aumento (figura 2).

\section{Colesterol HDL}

Se encontraron diferencias estadísticamente significativas entre las concentraciones de colesterol HDL de los tratamientos en todas las semanas. En la semana 1 las diferencias se observaron entre el grupo control y el tratamiento

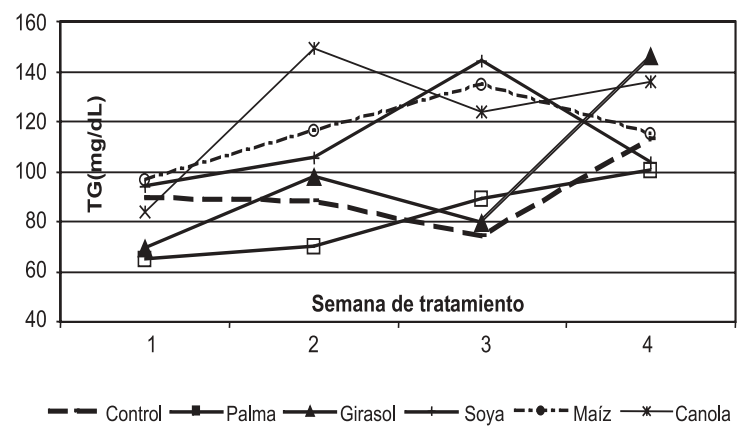

Figura 1. Concentraciones media de triglicéridos en las diferentes semanas de suplemento y según tipo de tratamiento con aceites vegetales. 
Cuadro 2. Concentraciones de triglicéridos, colesterol total y colesterol HDL según las semanas y tipo de suplemento con aceites vegetales.

\begin{tabular}{|c|c|c|c|c|c|c|c|c|}
\hline \multirow[b]{2}{*}{ Semana } & \multirow[b]{2}{*}{$\begin{array}{l}\text { Control } \\
(x \pm D E)\end{array}$} & \multirow[b]{2}{*}{$\begin{array}{l}\text { Palma } \\
(x \pm D E)\end{array}$} & \multirow[b]{2}{*}{$\begin{array}{l}\text { Girasol } \\
(x \pm D E)\end{array}$} & \multicolumn{2}{|c|}{ Tipo de tratamiento } & \multirow[b]{2}{*}{$\begin{array}{l}\text { Canola } \\
(x \pm D E)\end{array}$} & \multirow[b]{2}{*}{$\begin{array}{l}\text { Valor de p } \\
\text { (Levene) }\end{array}$} & \multirow[b]{2}{*}{$\begin{array}{r}\text { Valor de } \mathrm{P} \\
\text { (ANOVA) }\end{array}$} \\
\hline & & & & $\begin{array}{c}\text { Soya } \\
(x \pm D E)\end{array}$ & $\begin{array}{c}\text { Maíz } \\
(x \pm D E)\end{array}$ & & & \\
\hline \multicolumn{9}{|c|}{ Triglicéridos (mg/dL) } \\
\hline 1 & $89,5 \pm 59,8$ & $64,5 \pm 6,4$ & $69,0 \pm 14,7$ & $93,8 \pm 39,9$ & $96,5 \pm 26,6$ & $83,5 \pm 42,3$ & 0,033 & 0,749 \\
\hline 2 & $88,0 \pm 35,2$ & $69,8 \pm 3,3$ & $97,5 \pm 54,8$ & $105,5 \pm 66,0$ & $116,5 \pm 37,4$ & $149,5 \pm 49,3$ & 0,393 & 0,268 \\
\hline 3 & $74,0 \pm 30,8$ & $88,8 \pm 16,2$ & $79,3 \pm 14,3$ & $144,0 \pm 34,3$ & $134,5 \pm 34,7$ & $123,8 \pm 40,2$ & 0,622 & $0,009^{*}$ \\
\hline 4 & $112,8 \pm 28,9$ & $100,5 \pm 27,6$ & $146,0 \pm 28,3$ & $103,5 \pm 30,6$ & $114,8 \pm 59,0$ & $135,8 \pm 45,9$ & 0,474 & 0.510 \\
\hline \multicolumn{9}{|c|}{$\begin{array}{l}\text { Kruskall-Wallis para triglicéridos, primera semana: } P=0,439 \\
\text { Bonferroni, primera semana: control-soya }(P=0,038)^{*}\end{array}$} \\
\hline \multicolumn{9}{|c|}{ Colesterol total $(\mathrm{mg} / \mathrm{dl})$} \\
\hline $\begin{array}{l}1 \\
2 \\
3 \\
4\end{array}$ & $\begin{array}{l}84,8 \pm 4,5 \\
65,5 \pm 11.8 \\
72,8 \pm 10,0 \\
67,5 \pm 12,7\end{array}$ & $\begin{array}{c}91,3 \pm 8,7 \\
71,3 \pm 5,6 \\
75,3 \pm 8,9 \\
78,8 \pm 16,2\end{array}$ & $\begin{array}{c}71,3 \pm 8,7 \\
76,3 \pm 12,3 \\
78,0 \pm 6,2 \\
72,5 \pm 11,9\end{array}$ & $\begin{array}{c}78,3 \pm 7,9 \\
91,3 \pm 30,0 \\
71,5 \pm 4,5 \\
76 \pm 6,1\end{array}$ & $\begin{array}{c}84,8 \pm 5,0 \\
90,0 \pm 16,7 \\
86 \pm 10,3 \\
82,5 \pm 10,5\end{array}$ & $\begin{array}{c}85,0 \pm 13,0 \\
77,3 \pm 5,1 \\
79,0 \pm 10,6 \\
85,8 \pm 7,3\end{array}$ & $\begin{array}{l}0,054 \\
0,117 \\
0,751 \\
0,232\end{array}$ & $\begin{array}{l}0,055 \\
0,199 \\
0,242 \\
0,272\end{array}$ \\
\hline \multicolumn{9}{|c|}{ Kruskall-Wallis para colesterol total, primera semana: $P=0,011$} \\
\hline \multicolumn{9}{|c|}{ Colesterol HDL (mg/dl) } \\
\hline $\begin{array}{l}1 \\
2 \\
3\end{array}$ & $\begin{array}{c}31,8 \pm 2,1 \\
24 \pm 0,8 \\
28,2 \pm 1,3\end{array}$ & $\begin{array}{l}30,3 \pm 3,9 \\
27,5 \pm 1,3 \\
26,8 \pm 1,0\end{array}$ & $\begin{array}{l}25,5 \pm 0,6 \\
27,8 \pm 2,1 \\
29,8 \pm 1,3\end{array}$ & $\begin{array}{l}28,8 \pm 1,0 \\
29,3 \pm 1,5 \\
24,5 \pm 0,6\end{array}$ & $\begin{array}{c}29,8 \pm 1,3 \\
32,5 \pm 1,7 \\
28,5 \pm 1,3\end{array}$ & $\begin{array}{l}31,5 \pm 1,3 \\
27,8 \pm 1,3 \\
24,8 \pm 1,0\end{array}$ & $\begin{array}{l}0,005 \\
0,404 \\
0,769\end{array}$ & $\begin{array}{l}0,004^{*} \\
0,000^{*} \\
0,000^{*}\end{array}$ \\
\hline 4 & $32,8 \pm 1,3$ & $26,5 \pm 1,3$ & $31,5 \pm 0,58$ & $26 \pm 0,8$ & $32,8 \pm 1,3$ & $31,5 \pm 0,6$ & 0,342 & $0,000^{*}$ \\
\hline \multicolumn{9}{|c|}{$\begin{array}{l}\text { Bonferroni para C-HDL } \\
\text { Semana 1: control-girasol }(p=0,005)^{\star} / \text { palma-girasol }(p=0,05)^{\star} / \text { canola-girasol }(p=0,007)\end{array}$} \\
\hline \multirow{2}{*}{\multicolumn{9}{|c|}{ 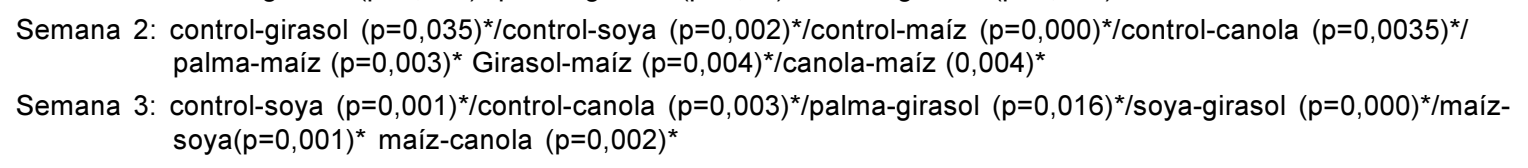 }} \\
\hline & & & & & & & & \\
\hline \multicolumn{9}{|c|}{ Semana 4: Control-palma/palma-maíz/palma-canola/girasol-palma/girasol-soya/control-soya/soya-maíz $(p=0,000)^{*}$} \\
\hline
\end{tabular}

*Diferencias significativas

con girasol, palma-girasol y canola-girasol; en todos los casos, el grupo con suplemento de aceite de girasol presentó las menores concentraciones de colesterol HDL y el grupo control y de canola, las mayores concentraciones (cuadro 2).

En la semana 2 las diferencias se presentaron entre el grupo control versus el de girasol, control versus aceite de soya, control versus maíz, control versus canola, palma y maíz, y girasol y maíz y canola versus maíz; el grupo control obtuvo las menores concentraciones y el grupo con suplemento de aceite de maíz mostró las mayores concentraciones de colesterol HDL, seguido por los grupos de soya, girasol, canola y palma (cuadro 2).

En la semana 3 se percibieron diferencias significativas específicamente entre el grupo control y los de tratamientos de aceite de soya y canola, con las menores concentraciones de colesterol HDL en los dos grupos con tratamiento. También se observaron diferencias entre los grupos de palma versus girasol, soya versus girasol, con mayores concentraciones de colesterol HDL en el grupo con suplemento de girasol, maíz versus soya y maíz versus canola con las mayores concentraciones en el grupo del maíz (cuadro 2).

En la semana 4 se observaron de nuevo diferencias significativas entre los tratamientos, particularmente entre los grupos control-palma, palma-maíz, palma-canola, girasol-palma, girasolsoya, rodentina-soya y soya-maíz; el grupo control y los tratamientos de girasol, maíz y canola mantuvieron un efecto semejante y diferente al de palma y soya que exhibieron menores concentraciones de colesterol HDL (cuadro 2). 


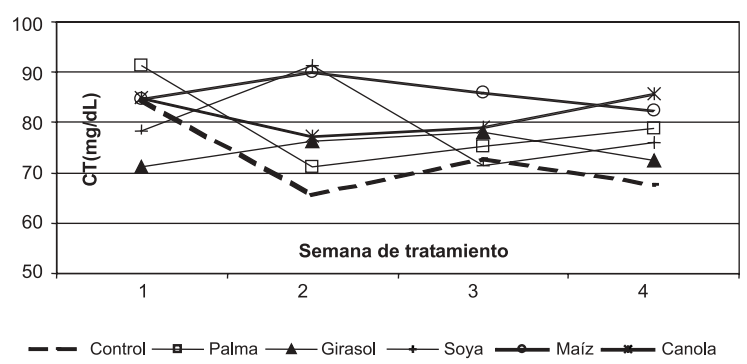

Figura 2. Concentraciones media de colesterol total en las diferentes semanas de suplemento y según el tipo de tratamiento con aceites vegetales.

La figura 3 muestra la tendencia de los valores medio de las concentraciones colesterol HDL en las diferentes semanas y tratamientos; se observó una tendencia a la disminución de las concentraciones de colesterol HDL en el grupo con suplemento de aceite de palma y una tendencia al aumento en el grupo con el suplemento de aceite de girasol y maíz. En el grupo con suplemento de aceite de girasol, las concentraciones de colesterol HDL mostraron inicialmente un valor de $25,5 \mathrm{mg} / \mathrm{dl}$ en la primera semana y, después de 4 semanas, aumentó linealmente hasta una concentración de $31,5 \mathrm{mg} /$ dl; el grupo de maíz empezó con una mayor concentración que la del girasol $(29,8 \mathrm{mg} / \mathrm{dl})$, aumentó en la segunda semana, disminuyó en la tercera y volvió a aumentar en la cuarta semana hasta un valor mayor al de los otros grupos de tratamiento pero igual al observado en el grupo control $(32,8 \mathrm{mg} / \mathrm{dl})$. El grupo con aceite de palma empezó con una concentración de $30,3 \mathrm{mg} / \mathrm{dl} \mathrm{y}$ para la cuarta semana había disminuido a 26,5 $\mathrm{mg} / \mathrm{dl}$ (cuadro 2, figura 3).

\section{Discusión}

Los resultados muestran que las diferencias en la composición de los aceites sí pueden afectar de forma positiva o negativa el perfil lipídico de los animales y que las respuestas en cada variable lipídica difieren según la composición de los aceites. En las concentraciones de triglicéridos, los grupos no presentaron diferencias estadísticamente significativas a excepción del grupo control y de soya en la tercera semana de tratamiento; no obstante, en el grupo con suplemento de aceite de palma se observó una tendencia a las menores concentraciones para esta variable durante cada semana de tratamiento y en los grupos con suplementos de girasol y canola, una tendencia al aumento. Este efecto coincide con otros estudios que explican que el contenido de tocoferoles, tocotrienoles y betacarotenos disminuye los triglicéridos a pesar de que exista alto contenido de ácidos grasos saturados (15-16). En este estudio, el aceite de palma presentó la mayor concentración de ácidos grasos saturados, pero también alto contenido de tocotrienoles y el único con $\delta$-tocotrienol; además, un alto contenido de ácidos grasos monoinsaturados que puede ser otra de las razones que expliquen su efecto en los triglicéridos $(20,21)$.

La tendencia a mayores concentraciones de triglicéridos en los aceites de girasol y de canola puede estar relacionada con las bajas concentraciones de vitamina $E$ que, en alguna forma, puede atenuar el efecto positivo que se le atribuye en algunos estudios al alto contenido de ácidos grasos poli y monoinsaturados $y$, especialmente, al contenido de $\alpha$-linoleico (17-19).

Se observó variabilidad en los valores de triglicéridos tanto en las ratas con diferentes tratamientos como en las que se utilizaron como control; esta variabilidad podría tener relación con el periodo de crecimiento de las ratas que incide en los lípidos (1-3); por ello, sería conveniente manejar ratas adultas jóvenes en futuras investigaciones.

Teniendo en cuenta que en nuestro medio la hipertrigliceridemia es muy prevalente y que

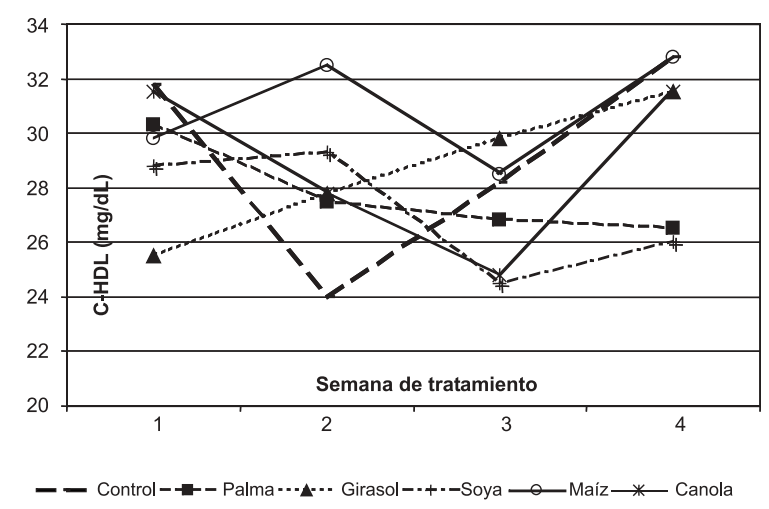

Figura 3. Concentraciones media de colesterol HDL en las diferentes semanas de suplemento y según el tipo de tratamiento con aceites vegetales. 
Colombia es uno de los mayores productores de aceite de palma, sería relevante realizar estudios para evaluar su efecto en otros modelos animales o en humanos con hipertrigliceridemia y estudiar también los efectos de los aceites de canola y de girasol en los triglicéridos después de ser enriquecidos con vitamina $E$, especialmente, en forma de tocotrienol.

En cuanto a las concentraciones de colesterol total no se encontraron diferencias significativas entre los tratamientos en ninguna de las semanas de intervención. Los resultados son contrarios a los estudios que mencionan que una dieta con suplemento de tocoferoles y tocotrienoles reduce el colesterol total (3-5). Sin embargo, el efecto probablemente se relaciona con el tiempo de exposición al suplemento y al contenido administrado de lípidos; se sugiere desarrollar ensayos con mayor tiempo de exposición al suplemento y aumentar el porcentaje de grasa que se administra. Barbosa et al. mencionan que los lípidos de las ratas cambian con una dieta 3 a 10 veces mayor en grasa que el requerimiento normal (8).

En el colesterol HDL se encontraron diferencias estadísticamente significativas entre grupos y en todas las semanas de tratamiento. Estos resultados ratifican la idea de que el colesterol HDL puede ser más sensible a los cambios por el efecto de los diferentes tipos de aceites (8). Se observó, además, una tendencia a la disminución en las concentraciones de colesterol HDL en el grupo con suplemento de aceite de palma y una tendencia al aumento en el grupo con suplemento de aceite de girasol y maíz; este efecto, posiblemente, se relaciona con la baja proporción de ácidos grasos saturados en estos dos aceites versus el alto contenido en el aceite de palma y la mayor proporción de $\alpha$-tocoferol en el aceite de girasol; la última deducción se correlaciona con los estudios que le atribuyen al $\alpha$-tocoferol un efecto protector contra la oxidación del colesterol LDL y contra la placa ateromatosa (9-11); estos efectos pueden guardar relación con los cambios en las concentraciones de colesterol HDL.

Con el aceite de canola no se encontró ningún efecto favorable sobre las concentraciones del perfil lípidico, resultado contrario a otros estudios que mencionan modificaciones en el perfil lipídico de las ratas debido a su bajo contenido de ácidos grasos saturados y alto contenido de ácidos grasos mono y poliinstaurados $(8,17-21)$.

En conclusión, se observó que en este modelo animal no hay efectos de los aceites vegetales sobre el colesterol total, pero sí en los triglicéridos y en el colesterol HDL; éste es el más susceptible a cambios, con disminución en las concentraciones del grupo con suplemento de aceite de palma y aumento en el grupo con suplemento de aceite de girasol y maíz. Los tocotrienoles del aceite de palma, al parecer, ejercen algún efecto de disminución en las concentraciones de triglicéridos, por lo cual es pertinente evaluar este efecto en otros modelos animales, especialmente de hipertrigliceridemia y sopesarlo con el efecto negativo que probablemente tiene sobre el colesterol HDL.

Los aceites de girasol y canola mostraron cambios desfavorables sobre las concentraciones de triglicéridos de las ratas. Sin embargo, se requieren otros estudios para confirmar estos hallazgos; sería relevante, además, estudiar la acción del aceite de girasol en los triglicéridos, enriqueciéndolo con tocotrienoles; lo anterior si se tiene en cuenta que, al parecer, modula una respuesta favorable sobre el colesterol HDL y, por lo tanto, su enriquecimiento con este compuesto podría aumentar su potencial para disminuir el riesgo cardiovascular.

Al considerar los efectos positivos del aceite de maíz sobre el colesterol HDL de las ratas, su bajo contenido de ácidos grasos saturados, la alta proporción de ácidos grasos mono y poliinsaturados, $\alpha$-tocoferol y tocotrienol, se debería aumentar su contenido de tocotrienoles y evaluar si genera cambios favorables en otras variables lipídicas como en los triglicéridos.

Los resultados en este modelo animal muestran que el bajo contenido de ácidos grasos saturados en los aceites parece ser importante para aumentar las concentraciones de colesterol HDL; los tocoferoles, posiblemente, mejoran la respuesta para aumentar el colesterol HDL en aceites ricos en ácidos grasos monoinsaturados y poliinsaturados, y los tocotrienoles podrían 
contribuir a disminuir los triglicéridos y a disminuir las respuestas desfavorables en el perfil lipídico que se han observado con los ácidos grasos saturados. No obstante, estas hipótesis requieren comprobación con otras investigaciones.

\section{Agradecimientos}

A Colciencias y al personal del Bioterio del Instituto Nacional de Salud por su colaboración en la fase in vivo del proyecto.

\section{Conflicto de intereses}

Los resultados de esta investigación no representan ningún tipo de conflicto por intereses particulares de las instituciones participantes, excepto, aquéllos relacionados con la disposición por generar o apoyar la búsqueda de conocimiento científico.

\section{Financiación}

El proyecto fue financiado conjuntamente por Colciencias (proyecto 21040412688), el Instituto Nacional de Salud, Cenipalma y la Pontificia Universidad Javeriana.

\section{Referencias}

1. Herrera E, Barbas C. Vitamin E: action, metabolism and perspectives. J Physiol Biochem 2001;1:43-56.

2. Evstigneeva R. Volkov I.M, Chudinova V. Vitamin E as a universal antioxidant and stabilizer of biological membranes. Memb Cell Biol 1998;2:151-72.

3. Brigelius-Flohe R, Traber MG. Vitamin E: function and metabolism. FASEB J 1999;13:1145-55.

4. Kaul N, Devaraj S, Jialal I. Tocopherol and atherosclerosis. Exp Biol Med 2001;226:5-12.

5. Qureshi AA, Qureshi N, Wright JJ, Shen Z, Kramer G, Gapor A. Lowering of serum cholesterol in hypercholesterolemic humans by tocotrienols (palmvitee). Am J Clin Nutr 1991;53(Suppl.4):1021S-6S.

6. Febles C, Soto C, Saldaña A, García B. Funciones de la vitamina E. Rev Cubana Estomatol 2002;1:28-32.

7. Morris D. Canola and vitamin E. Canola Council of Canada. http://www.canola-council.org/pubs vitamin_E_ spanish.pdf. Fecha de consulta: marzo de 2004.

8. Barbosa Aguila M, Cota Loureiro C, Da Rocha Pinheiro A, De Lacerda Mandarim C. Lipid metabolism in rats fed diets containing different types of lipids Arq Bras Cardiol 2002;1:32-8.

9. Williams RJ, Motteram JM, Sharp $\mathbf{C H}$, Gallagher PJ. Dietary vitamin E and attenuation of early lesion development in modified Watanabe rabbits. Atherosclerosis 1992;94:153-9.

10. Verlangieri A, Buxh M. Effects of $\alpha$-tocopherol supplementation on experimentally induced primate atherosclerosis. J Am Coll Nutr 1992;11:131-8.

11. Prasad J. Effect of vitamin $E$ supplementation on leukocyte function. Am J Clin Nutr 1980;33:606-8.

12. Qureshi AA, Sami SA, Salser WA, Khan FA. Dosedependent suppression of serum cholesterol by tocotrienol-rich fraction (TRF25) of rice bran in hypercholesterolemic humans. Atherosclerosis 2002; 1:199-207

13. Raederstorff D, Elste V, Aebischer C, Weber P. Effect of either gamma-tocotrienol or a tocotrienol mixture on the plasma lipid profile in hamsters. Ann Nutr Metab 2002;1:17-23.

14. Hayes KC, Pronczuk A, Liang JS. Differences in the plasma transport and tissue concentrations of tocopherols and tocotrienols: observations in humans and hamsters. Proc Soc Exp Biol Med 1993;3:353-9.

15. Chong YH, Ng TK. Effects of palm oil on cardiovascular risk. Med J Malaysia 1991;1:41-50.

16. Ong AS, Goh SH. Palm oil: a healthful and cost-effective dietary component. Food Nutr Bull 2002;1:11-22.

17. McLennan PL, Dallimore JA. Dietary canola oil modifies myocardial fatty acids and inhibits cardiac arrhythmias in rats. J Nutr 1995;125:1003-9.

18. McLennan PL. Relative effects of dietary saturated, monounsaturated and polyunsaturated fatty acids on cardiac arrhythmias in rats. Am J Clin Nutr 1993;57:20712.

19. Baba NH, Antoniades K, Habbal Z. Effects of dietary canola, olive and linolenic acid enriched olive oils on plasma lipids, lipid peroxidation and lipoprotein lipase activity in rats. Nutr Res 1999;19:601-12.

20. Mattson FH, Grundy SM. Comparison of the effects of dietary saturated, monounsaturated and polyunsaturated fatty acids on plasma lipids and lipoproteins in man. J Lipids Res 1985;26:194.

21. Stone NJ. Fish consumption, fish oil, lipids, and coronary heart disease. Circulation 1996;23:37-40.

22. Campbell SE, Gerdes AM, Smith TD. Comparison of regional differences in cardiac cardiomyocite dimensions in rats, hamsters, and guinea pigs. Anat $\operatorname{Rec} 1986 ; 219: 53-9$. 